\title{
Stability and control of power grids with diluted network topology
}

Cite as: Chaos 29, 123105 (2019); https://doi.org/10.1063/1.5111686

Submitted: 29 May 2019. Accepted: 14 November 2019. Published Online: 09 December 2019

Liudmila Tumash (D), Simona Olmi (iD, and Eckehard Schöll (D)

\section{COLLECTIONS}

Paper published as part of the special topic on Dynamics of Modern Power Grids

Note: This paper is part of the Focus Issue on the Dynamics of Modern Power Grids.
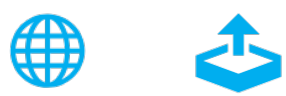

\section{ARTICLES YOU MAY BE INTERESTED IN}

Simulating waves, chaos and synchronization with a microcontroller

Chaos: An Interdisciplinary Journal of Nonlinear Science 29, 123104 (2019); https:// doi.org/10.1063/1.5094351

\section{Cluster synchronization: From single-layer to multi-layer networks}

Chaos: An Interdisciplinary Journal of Nonlinear Science 29, 123120 (2019); https://

doi.org/10.1063/1.5122699

\section{Multistability in lossy power grids and oscillator networks}

Chaos: An Interdisciplinary Journal of Nonlinear Science 29, 123119 (2019); https://

doi.org/10.1063/1.5122739

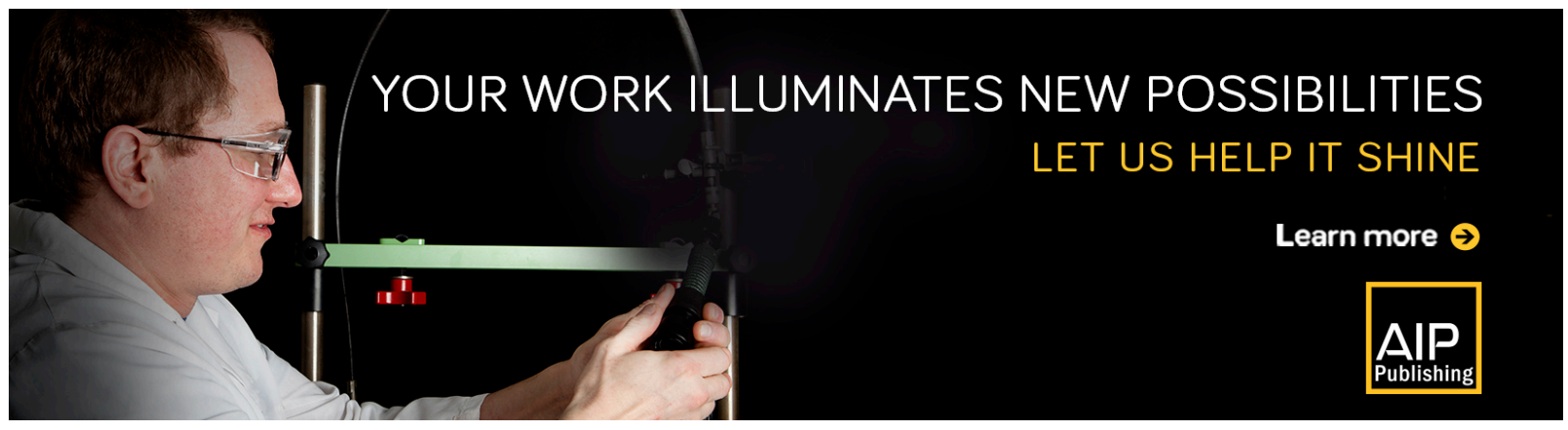




\title{
Stability and control of power grids with diluted network topology
}

\author{
Cite as: Chaos 29, 123105 (2019); doi: 10.1063/1.5111686 \\ Submitted: 29 May 2019 . Accepted: 14 November 2019. \\ Published Online: 9 December 2019

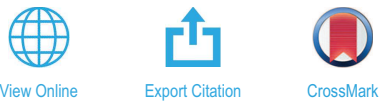

Liudmila Tumash, ' (D) Simona Olmi, ${ }^{2,3, a)}$ (I) and Eckehard Schöll'

\begin{abstract}
AFFILIATIONS
1 Institut für Theoretische Physik, Technische Universität Berlin, Hardenbergstraße 36, 10623 Berlin, Germany

${ }^{2}$ INRIA Sophia Antipolis Méditerranée, 2004 Route des Lucioles, 06902 Valbonne, France

${ }^{3} \mathrm{CNR}$-Consiglio Nazionale delle Ricerche_-Istituto dei Sistemi Complessi, 50019 Sesto Fiorentino, Italy
\end{abstract}

Note: This paper is part of the Focus Issue on the Dynamics of Modern Power Grids.

a) Electronic mail: simona.olmi@fi.isc.cnr.it

\begin{abstract}
We consider sparse random networks of Kuramoto phase oscillators with inertia in order to mimic and investigate the dynamics emerging in high-voltage power grids. The corresponding natural frequencies are assumed to be bimodally Gaussian distributed, thus modeling the distribution of both power generators and consumers, which must be in balance. Our main focus is on the theoretical analysis of the linear stability of the frequency-synchronized state, which is necessary for the stable operation of power grids and the control of unstable synchronous states. We demonstrate by numerical simulations that unstable frequency-synchronized states can be stabilized by feedback control. Further, we extend our study to include stochastic temporal power fluctuations and discuss the interplay of topological disorder and Gaussian white noise for various model configurations and finally demonstrate that our control scheme also works well under the influence of noise. Results for synthetic Erdös-Renyi random networks with low average connectivity and with symmetric or asymmetric bimodal frequency distributions are compared with those obtained by considering a real power grid topology, namely, the grid of Italy.
\end{abstract}

Published under license by AIP Publishing. https://doi.org/10.1063/1.5111686

The goal of this paper is to investigate complex dynamic networks that can model high-voltage power grids with renewable, fluctuating energy sources. For this purpose, we use the Kuramoto model of phase oscillators with inertia (rotators) to model the network of power generators and consumers. In particular, we analyze the synchronization transition of networks of $N$ phase oscillators, whose natural frequencies are bimodally distributed, corresponding to the distribution of generator and consumer power. Moreover, we take into account random networks and Gaussian white noise in order to mimic the topological disorder and temporal power fluctuations, respectively. Results for synthetic randomly diluted networks are then compared with those obtained by considering a real power grid topology, i.e., the grid of Italy. Our main focus is on the stability of the synchronized state, which is necessary for the stable operation of power grids and its control. We present numerical simulations of the deterministic and stochastic dynamics and a theoretical analysis of the stability against small perturbations and show that unstable synchronous states can be stabilized by a control loop. Further, we study the influence of noise, which is typical for renewable energy sources, and its interplay with topological disorder, both for sparse synthetic random networks and for the Italian power grid.

\section{INTRODUCTION}

Within the last century, electrical energy has been generated mainly by power plants based on coal or nuclear power. ${ }^{1}$ Nowadays, we are witnessing a time of drastic changes in the operation of power grids due to the necessity to reduce global warming caused by a large emission of carbon dioxide gases. ${ }^{2,3}$ In particular, the generating units of a power grid are more and more supplied by natural sources, such as wind parks, photovoltaic arrays, and other renewable energy sources. ${ }^{4}$ The main question here remains the sustainable and stable operation of power grids, which is of vital importance to our daily lives. ${ }^{5-8}$ However, due to the regime shift toward renewable energy sources, three major changes have to be envisaged in modern power grids. The first change is decentralization, i.e., the power system represents a distributed network carrying many small units of energy to the consumers instead of large units of energy coming 
from a few power plants. ${ }^{9,10}$ The second change is a strong spatial separation between power generators and consumers. ${ }^{11}$ It is evident that power systems based on solar or wind energy should be located in areas where such energy is abundant. Finally, the last important change is the increasing fraction of strongly fluctuating power output due to renewable energy sources, which are strongly dependent on weather conditions. ${ }^{12-18}$

The investigation of power grid systems has been recently addressed from a nonlinear dynamics point of view, using the Kuramoto phase oscillator model with inertia. ${ }^{19-30}$ The modification of the Kuramoto model by an additional inertial term was first reported and investigated by Tanaka, Lichtenberg, and Oishi, ${ }^{31,32}$ who were inspired by Ermentrout ${ }^{33}$ when choosing a phase oscillator model able to show a transition to synchronization via frequency adaptation instead of the usual phase-locking mechanism. That work specifically focused on a phase oscillator model able to mimic the synchronization mechanism observed in the firefly Pteroptix malaccae. ${ }^{33}$ Recently, the model has been used to investigate the self-synchronization emerging in disordered arrays of underdamped Josephson junctions $^{34}$ as well as to show the emergence of explosive synchronization ${ }^{35}$ in a network of rotators whenever the natural frequency is chosen to be proportional to the node degree. Nowadays, the Kuramoto model with inertia is a standard mathematical model used to study the dynamical behavior of power generators and consumers ${ }^{19-27}$ since it captures the essential dynamical features of a power grid on coarse scales but is still simple enough to allow for a comprehensive understanding of the fundamental properties of power grid dynamics.

The Kuramoto model with inertia includes an additional term that takes into account the frequency dynamics. Oscillators are able to reach frequency synchronization by adapting themselves to some intrinsic collective frequency. In particular, power grids tend to synchronize their frequencies to the standard ac power frequency of $\Omega=50 \mathrm{~Hz}$ (or $60 \mathrm{~Hz}$ in some countries). The description of highvoltage power grids in terms of the extended Kuramoto model has been first proposed by Filatrella et al., ${ }^{19}$ who distinguished the power generated by power sources $\left(P_{\text {source }}^{i}>0\right)$ from the power consumed by passive machines or loads $\left(P_{\text {cons }}^{i}<0\right)$. Such a power separation implies using a bimodal distribution of the power (corresponding to the dimensionless natural frequencies in the dimensionless Kuramoto model) within the network. Although this is a very important feature of the model, most of the previous studies consider either a unimodal frequency distribution ${ }^{24,36}$ or $\delta$-function shaped bimodal distributions. ${ }^{22,23,36}$ In our work, we use a bimodal Gaussian distribution of frequencies, which models consumed and generated power in a more realistic way. Moreover, from the topological viewpoint, we focus on randomly diluted networks, giving rise to a more realistic description of power grids than all-to-all coupled networks, since real-world systems are characterized by low connectivity per node. As a proof of principle, results are compared with those obtained by considering the real Italian high-voltage power grid, supplemented by a bimodal $\delta$-distribution of natural frequencies.

The stable operation of power grids is characterized by maintaining a synchronous state of the entire network. The present paper focusses on the stability and control of frequency-synchronized solutions emerging in sparse populations of $N$ Kuramoto oscillators with inertia (rotators). A detailed stability analysis of a population of $N$ rotators, randomly connected, is reported in order to clarify how stable and unstable solutions can be found before applying the control technique. A similar stability analysis was performed by Mirollo et al..$^{37}$ and by Delabays et al. ${ }^{38}$ for networks of classical Kuramoto oscillators (without inertia) with different topologies (fully coupled networks and planar graphs, respectively). Regarding networks of rotators, a stability analysis has been presented in Refs. 25 and 39-41 for globally coupled networks and chain structures. In particular, Coletta et al. ${ }^{40}$ have extended the stability analysis performed by Manik et al. ${ }^{39}$ for networks with inhomogeneous damping but identical inertia to the case of inhomogeneous inertia and damping. Here, we use the stability analysis to characterize unstable synchronous states in diluted networks, which we subsequently stabilize by a control loop.

The present article is structured as follows. In Sec. II, the model and the dynamical indicators are presented. After discussing the onset of synchronization in a randomly diluted network (Sec. III) and characterizing the frequency-synchronized solution (Sec. IV), we analytically establish the criteria for the stability of the synchronous state (Sec. V), and we numerically solve the eigenvalue problem of $N$ coupled rotators by using the Levenberg-Marquardt algorithm $^{42-44}$ (Sec. VI). This allows us to obtain, for all $N$ rotators, a set of initial phases for which a frequency-synchronized solution evolves. Moreover, we determine the minimum coupling strength for which frequency synchronization is still possible and we derive both stable and unstable solutions, as illustrated in Sec. VI, where solutions are shown as the spatiotemporal evolution of state variables. In Sec. VII, we apply a control method to stabilize the unstable frequency-synchronized solutions. Thus, control enables us to realize a stable synchronous state even if this does not exist in the uncontrolled system for too small coupling constant or nonzero initial phase differences. In Sec. VIII, we add temporal random fluctuations and consider the interplay of Gaussian white noise and spatial disorder due to diluted connectivities. We examine how the frequencysynchronized solution changes with respect to the deterministic case, taking into account different setups (i.e., different frequency distributions and different topologies). Finally, in Sec. IX, the control is applied to the stochastic setups investigated in Sec. VIII. The results are discussed in Sec. X, while, in the Appendix, further deterministic setups are considered demonstrating the wide applicability of our control method.

\section{MODEL}

The investigated system consists of a population of $i=1, \ldots, N$ coupled Kuramoto oscillators with inertia and reads

$$
m \ddot{\theta}_{i}+\dot{\theta}_{i}=\Omega_{i}+\frac{K}{N_{i}} \sum_{j=1}^{N} A_{i j} \sin \left(\theta_{j}-\theta_{i}\right),
$$

where $\theta_{i}$ and $\dot{\theta}_{i}$ are the instantaneous phase and frequency, respectively, of oscillator $i$. In terms of the power grid, $\dot{\theta}_{i}$ represents the angular velocity of the $i$ th oscillator, which corresponds to the frequency deviation, divided by $2 \pi$, with respect to the standard grid frequency $(50$ or $60 \mathrm{~Hz}$ ). The parameter $m>0$ indicates the inertial mass of generators and loads that plays a fundamental role in determining the hysteretic transition to synchronization. $K>0$ is 
the coupling constant of the network, which measures the strength of the connectivities among the oscillators. In terms of power grid systems, $K$ is equivalent to the transmission line capacities between loads and generators. Increasing coupling strength enhances the synchronization of elements with heterogeneous natural frequencies. $A$ is the connectivity matrix, whose entries $A_{i j}$ can be either one, provided that there is a link between nodes $i$ and $j$, or zero if the link is absent. From the topological viewpoint, the power grid network is an undirected, symmetric graph, i.e., $A_{i j}=A_{j i}$. $N_{i}$ is the node degree of the $i$ th element, thus denoting the number of the links outcoming from this node. Throughout this study, we will mainly consider Erdös-Renyi networks, i.e., the graph is constructed by connecting nodes randomly with a constant probability. This topology turns out to be more realistic in comparison to a globally coupled network, since power grid networks are characterized by only a few links per node. We assume a constant node degree $N_{i}=N_{c}$ and a dilution parameter $p=\frac{N_{c}}{N}$. The latter indicates the ratio of existing links to the number of all possible links. These so-called diluted networks are thus obtained by considering random realizations of the coupling matrix $A$, keeping the connectivity matrix symmetric. In order to understand the role played by topology in our analysis, we have also implemented a realistic power grid topology, i.e., the Italian high-voltage $(380 \mathrm{kV})$ power grid (Sardinia excluded), which is composed of $N=127$ nodes, divided into 34 sources (hydroelectric and thermal power plants) and 93 consumers, connected by 342 links. ${ }^{45}$

Finally, $\Omega_{i}$ represents the natural frequency of the oscillator $i$, whose value is chosen in accordance with a bimodal Gaussian distribution,

$$
g(\Omega)=\left[\frac{p_{g}}{\sqrt{2 \pi}} e^{-\frac{\left(\Omega-\Omega_{0^{+}}\right)^{2}}{2}}+\frac{1-p_{g}}{\sqrt{2 \pi}} e^{-\frac{\left(\Omega+\Omega_{0^{-}}\right)^{2}}{2}}\right] .
$$

In particular, $g(\Omega)$ is the superposition of two Gaussians with unit standard deviation, whose peaks are located at $\Omega_{0^{+}}$and $-\Omega_{0^{-}}$. Thus, the distance between the peaks is $\Omega_{0^{+}}+\Omega_{0^{-}}$. In the following, we will assume almost nonoverlapping Gaussians, with two different choices for the peak locations: (i) symmetric peak locations $\left(p_{g}=0.5, \Omega_{0^{+}}=\Omega_{0^{-}} \equiv \Omega_{0}=2\right)$ and (ii) asymmetric peak location to model the presence of fewer but larger generators and many small consumers $\left(p_{g}=0.2, \Omega_{0^{+}}=3.2, \Omega_{0^{-}}=0.8\right)$.

The physical motivation for choosing a bimodal distribution comes directly from Filatrella et al.: ${ }^{19}$ according to their work, each element of the power grid network either generates $\left(P_{\text {source }}^{i}>0\right)$ or consumes $\left(P_{\text {cons }}^{i}<0\right)$ power. Thus, one should distinguish two kinds of oscillators: the sources that deliver electrical power and the consumers that consume this power. Hence, the electrical power distribution of all oscillators should be bimodal, with a maximum at $P_{\text {source }}^{i}>$ 0 and one at $P_{\text {cons }}^{i}<0$. In the dimensionless Kuramoto model with inertia given by Eq. (1), this corresponds to a bimodal frequency distribution of $\Omega_{i}$ that we assume to be given by a superposition of two Gaussians with peaks at corresponding positive and negative frequencies. Thus, the necessary condition for the existence of the steady state is that the sum of the generated power equals the sum of the consumed power in order for the energy to be conserved. Note that, for the Italian high-voltage power grid, we have used a simplified formula for the bimodal distribution in order to make our findings comparable to previous studies. ${ }^{20,24}$ The distribution of $\Omega_{i}$ is composed of two $\delta$-functions located at $-\Omega_{0^{-}}=-1$ and $\Omega_{0^{+}}=2.7353$.
The phase ordering of the power grid is measured by the complex order parameter

$$
r(t) e^{i \phi(t)}=\frac{1}{N} \sum_{j=1}^{N} e^{i \theta_{j}},
$$

where its modulus $r(t) \in[0,1]$ and argument $\phi(t)$ indicate the degree of synchrony and mean phase angle, respectively. In the following, we will denote $r(t)$ as global order parameter, which corresponds to the standard Kuramoto order parameter. In the continuum limit, an asynchronous state is characterized by $r \approx 0$, while $r=1$ corresponds to full phase synchronization. Intermediate values of $r$ correspond to states with partial or cluster synchronization. It is worth noting that, for a phase-locked state, this parameter is not exactly 1 , but the state can be still synchronous whenever all frequencies are equal.

\section{DILUTED NETWORKS: THE ONSET OF SYNCHRONIZATION}

First, we explore the transition to synchronization for a randomly coupled set of power suppliers and consumers described by Eq. (1) with a symmetric bimodal frequency distribution. The random network we investigate is characterized by a dilution parameter of $p=0.20$, thus indicating that each node is randomly connected to $20 \%$ of all possible $N-1$ nodes. A typical synchronization transition profile is illustrated in Fig. 1(a), where we show the time-averaged global order parameter obtained by sweeping up and down adiabatically the connectivity strength $K$, following two different protocols as described in Refs. 24, 26, 31 and 28. In particular, protocol $(I)$ denotes the upsweep: the system's state variables $\left\{\theta_{i}\right\}$ and $\left\{\dot{\theta}_{i}\right\}=\left\{\omega_{i}\right\}$ are randomly initialized in the absence of coupling; then, the coupling strength is increased in steps of $\Delta K=0.5$ until the maximum coupling $K_{M}$ is reached (for $p=0.2$, we choose $K_{M}=60$ ). Note that the global order parameter increases as the elements become more strongly connected. Finally, it reaches the maximum value of $\bar{r} \approx 1$ as synchronization is achieved, which corresponds to the maximum coupling $K_{M}$. At each step, the initial conditions for phases and frequencies correspond to the final conditions obtained for the previous $K$ value. By protocol (II), we denote the reverse procedure: this time the initial state corresponds to the synchronized system at $K=K_{M}$, while the coupling is adiabatically decreased in steps $\Delta K=0.5$, until we approach again a completely uncoupled asynchronous system. For both protocols, the investigation of the nature of the dynamics emerging at each time step is done by using the same procedure: the system is simulated for a transient time $T_{R}$ followed by an investigation period $T_{W}$, during which the average values of global order parameter $\bar{r}$, the phase velocities $\left\{\bar{\omega}_{i}\right\}$, and the maximum natural frequency of the locked oscillators are calculated.

Now, we focus on a more detailed description of the different regimes emerging in the system by varying the coupling strength $K$, see Fig. 1(a). For small coupling constant, the system is uncoupled and asynchronous (AS), characterized by a low value of the time-averaged order parameter $\bar{r} \approx 1 / \sqrt{N}$ and nonidentical average phase velocities $\bar{\omega}_{i}$ for all the elements $i$, see Fig. 1(b). Increasing the coupling $K$ leads to a rapid jump of the average global order 

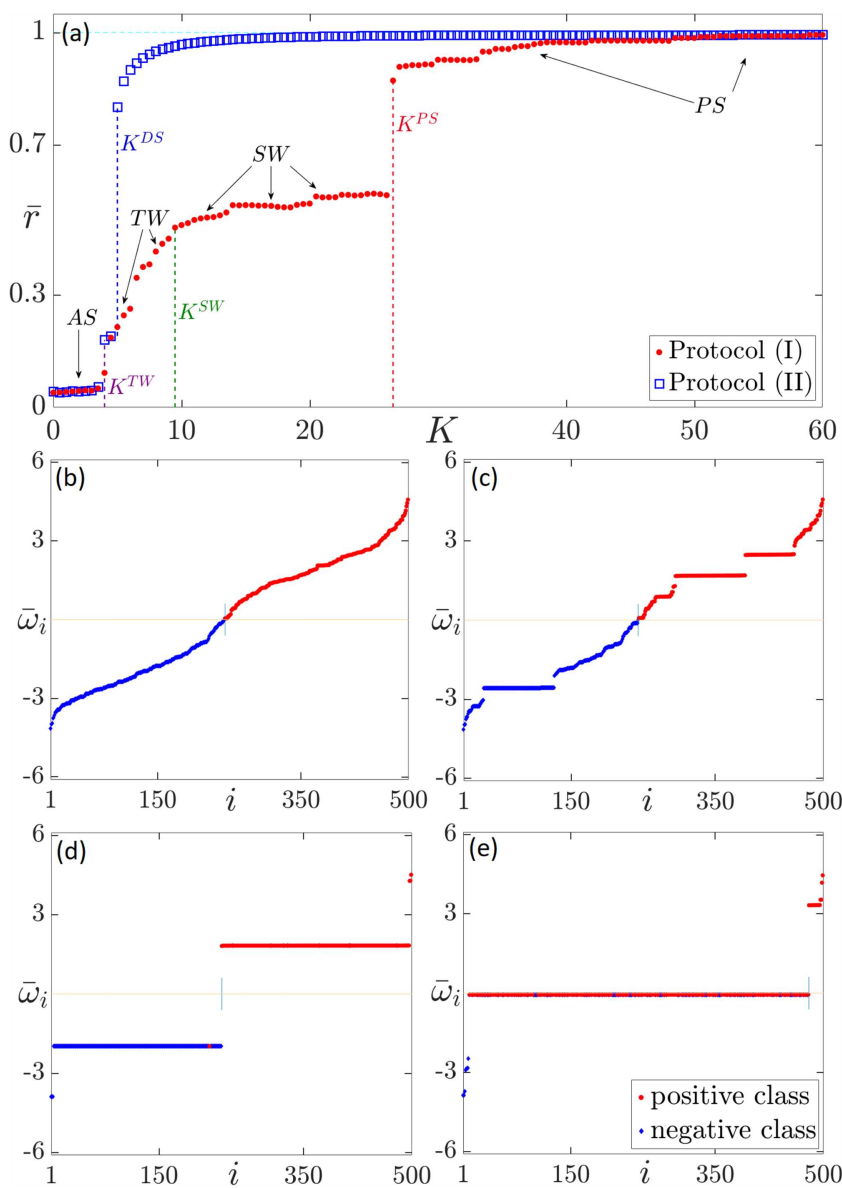

FIG. 1. (a) Time-averaged global order parameter $\bar{r}$ as a function of coupling constant $K$ for two series of simulations, obtained by following protocols (I) (upsweep, red filled circles) and (II) (downsweep, blue empty squares) for a diluted network with symmetric bimodal frequency distribution. The vertical dotted lines indicate the critical values of coupling $K$ for traveling waves $\left(K^{T W}\right.$, purple), standing waves $\left(K^{S W}\right.$, green), partial synchronization $\left(K^{P S}\right.$, red), and the value at which desynchronization occurs (KDS blue). Average phase velocity $\bar{\omega}_{i}$ as a function of node $i$ for (b) $K=2, \bar{r}=0.043$ (asynchronous state); (c) $K=5, \bar{r}=0.217$ (traveling wave); (d) $K=25, \bar{r}=0.567$ (standing wave); (e) $K=33, \bar{r}=0.928$ (partial synchronization). The nodes are labeled such that the average phase velocities $\bar{\omega}_{i}$ are sorted from low to high values. Positive (red) and negative (blue) classes refer to positive and negative natural frequency distributions, respectively. Parameters: $m=6, p=0.20, p_{g}=0.5, \Omega_{0}=2, N=500, T_{R}=4000, T_{W}=200$ $[\alpha=1 / 6, \sigma=\mathrm{K} /(\mathrm{pNm})=K / 600]$.

parameter $\bar{r}$ to higher values, i.e., $\bar{r}>0.1$. Here, we observe the emergence of one or more clusters of locked oscillators characterized by nodes with the same average phase velocity $\bar{\omega}_{i}$. The coexistence of chaotically whirling oscillators with clusters of locked nodes corresponds to a traveling wave (TW) solution [see Fig. 1(c)] that is observable for $K>K^{T W}$. A further increase of coupling can cause both the enlargement of the existing clusters of locked oscillators and the collapse of smaller clusters to larger ones, which are usually characterized by an average phase velocity $\bar{\omega}_{i} \approx \pm \Omega_{0}$. For $K>K^{S W}$, the system continuously approaches the standing wave state (SW), which is characterized by two symmetric clusters of locked oscillators drifting with opposite average phase velocities equal to $\bar{\omega}_{i} \approx \Omega_{0}$ and $-\Omega_{0}$ [see Fig. 1(d)]. In the SW regime, the system behaves like two independent subnetworks each one corresponding to a network with unimodal Gaussian frequency distribution, whose peaks are located respectively at $+\Omega_{0}$ (generators) or $-\Omega_{0}$ (loads). The corresponding time-averaged global order parameter equals $\bar{r} \approx 0.5$. Finally, for further increase of the coupling $K$, the average global order parameter $\bar{r}$ exhibits a rapid jump to higher values, i.e., $\bar{r}>0.9$. This means that for $K>K^{P S}$, the system reaches a partially (almost completely) synchronized regime. Thereby, the two subnetworks that for smaller $K$ behave almost independently, now merge into a unique stationary cluster with $\bar{\omega}_{i} \approx 0$, see Fig. 1(e). On the other hand, the number of unlocked oscillators is vanishingly small, i.e., $N-N_{L} \approx 0$. Such a rapid change of average global order parameter $\bar{r}$ allows us to identify the onset of synchronization of a network. As we continue increasing $K$, the system smoothly approaches the regime of full synchronization.

While the transition to synchronization for $K>K^{P S}$ is always detectable, irrespective of the chosen value of the dilution parameter $p$, the standing wave regime is not always detectable as the dilution increases and it actually disappears as the network topology becomes highly diluted, e.g., for $p<0.05$. In particular, as the randomness increases, it becomes more and more difficult for the system to reach such states as many elements will have different subgraphs of connected nodes with a variable percentage of nodes belonging to the same native class or to the opposite one, where the classes identify the oscillators with positive or negative natural frequencies, respectively. Therefore, the separation in two subnetworks with positive and negative classes, leading to a configuration similar to the one shown in Fig. 1(d) is hardly achieved. Finally, the disappearance of SW turns into a decrease of the critical value $K^{P S}$, as previously reported. ${ }^{28}$

If we analyze the system in accordance with protocol (II), the synchronous state survives for a large $K$ interval until it collapses toward asynchronicity at $K<K^{D S}$, where DS denotes desynchronization. Note that there is a considerable difference between the critical coupling values required to synchronize or desynchronize the system and $K^{P S}>K^{D S}$. In other words, the system needs a stronger coupling to become synchronized with respect to the desynchronizing value and once it is synchronized, due to inertia, it hardly leaves this regime. The transition to synchronization is, therefore, hysteretic and the size of the hysteresis loop $K^{P S}-K^{D S}$ depends on the inertia $m, K^{P S}$ is strongly affected by the dependence on $m .^{24,28}$

\section{SYNCHRONOUS SOLUTION}

Synchronization is a mandatory regime when the stable operation of power grids is required. Therefore, determining the stability of synchronous states is one of the central goals of the present study. In particular, we aim to investigate the stability of the synchronous solution emerging in a power grid network by linearizing the state around the frequency-synchronized solution, which gives a Jacobian matrix with constant coefficients, and analyzing the eigenvalues of this linearized system. The calculation of the eigenvalue with the largest real part $\lambda_{\max }$ will be the main criterion for determining the synchronization stability. Stability means that the sign of the largest real part is 
negative. If we rewrite Eq. (1) in terms of two dynamical variables, i.e., phase $\theta_{i}$ and frequency $\omega_{i}$, we obtain the following $2 N$-dimensional first-order system:

$$
\begin{aligned}
\dot{\theta}_{i} & =\omega_{i}, \\
\dot{\omega}_{i} & =\alpha\left(\Omega_{i}-\omega_{i}\right)+\sigma \sum_{j=1}^{N} A_{i j} \sin \left(\theta_{j}-\theta_{i}\right),
\end{aligned}
$$

where $\alpha=\frac{1}{m}$ and $\sigma=\frac{K}{N_{c} m}$.

Phase synchronization implies for all the phases $\theta_{1}=\theta_{2}=$ $\cdots=\theta_{c}$. If we denote the corresponding frequency as $\omega_{c}$, since the coupling term $\sigma \sum_{j=1}^{N} A_{i j} \sin \left(\theta_{j}-\theta_{i}\right)=0$, we obtain from Eq. (4)

$$
\dot{\omega}_{c}=\alpha\left(\Omega_{i}-\omega_{c}\right) \Rightarrow \Omega_{i}=\alpha^{-1} \dot{\omega}_{c}+\omega_{c},
$$

which holds only for the trivial case $\Omega_{i}=$ constant, since the right side of equation is not a function of $i$, while we have assumed a bimodal distribution for the natural frequencies. This suggests that complete phase synchronization is not achievable in our system. At this point, it is important to note that, in synchronous power grids, the phases must be different to enable the energy transmission, but they have a fixed and time-independent relation, i.e., they are locked. Thus, the fundamental state that enables stable operations in electrical grids is a frequency-synchronized synchronous state, characterized by phase-locking between the phases.

Moreover, in the case of frequency synchronization: $\dot{\theta}_{1}=\cdots=$ $\dot{\theta}_{n}=\omega_{c} \Rightarrow \theta_{i}-\theta_{j}=$ constant. If we rename the coupling term as $\chi_{i}=\sigma \sum_{j=1}^{N} A_{i j} \sin \left(\theta_{j}-\theta_{i}\right)$, from Eq. (4), we get

$$
\dot{\omega}_{c}=\alpha \Omega_{i}-\alpha \omega_{c}+\chi_{i}
$$

Note that variables $\dot{\omega}_{c}$ and $\omega_{c}$ do not depend on index $i$. Hence, we can define a constant $C_{0}$ such that

$$
\dot{\omega}_{c}+\alpha \omega_{c}=\alpha \Omega_{1}+\chi_{1}=\cdots=\alpha \Omega_{n}+\chi_{n}=C_{0} .
$$

By using the previous definition of $C_{0}$, we obtain a system of differential equations for the frequency-synchronized solution $\dot{\omega}_{c}=C_{0}-\alpha \omega_{c}$. If we assume to be in a frequency-synchronized regime $\left(\dot{\omega}_{c}=0\right)$, we can identify

$$
\omega_{c}=\frac{1}{\alpha} C_{0} .
$$

Finally, constant $C_{0}$ can be calculated by summing Eq. (6) over all nodes $i=1, \ldots, N$,

$$
\sum_{i=1}^{N}\left[\alpha \Omega_{i}-\alpha \omega_{c}+\sigma \sum_{j=1}^{N} A_{i j} \sin \left(\theta_{i}-\theta_{j}\right)=0\right] .
$$

Since the chosen network is a symmetric undirected graph, the term $\sum_{i=1}^{N} \sum_{j=1}^{N} A_{i j} \sin \left(\theta_{i}-\theta_{j}\right)=0$ and Eq. (8) reduces to

$$
\sum_{i=1}^{N} \alpha \Omega_{i}-\sum_{i=1}^{N} \alpha \omega_{c}=0 \Rightarrow \sum_{i=1}^{N} \alpha \Omega_{i}-N \alpha \omega_{c}=0 .
$$

Thus, it follows that for a finite system the value at which the frequency synchronizes is the arithmetic mean of all the natural frequencies,

$$
\omega_{c}=\frac{1}{N} \sum_{i=1}^{N} \Omega_{i}
$$

For a large network size, we expect this value to be close to 0 . In the present case, the arithmetic mean will be substituted with the expectation value, since natural frequencies are distributed according to a bimodal Gaussian distribution as shown in Eq. (2).

\section{STABILITY ANALYSIS OF FREQUENCY-SYNCHRONIZED SOLUTION}

The phase evolution of the system in the case of full frequency synchronization is given by

$$
\theta_{i}^{t}=\theta_{i}^{0}+\omega_{c} t
$$

where $\theta_{i}^{0}$ denotes the initial phase of oscillator $i$ at time $t=0$. Using the equality $\sin \left(\theta_{i}^{t}-\theta_{j}^{t}\right)=\sin \left(\theta_{i}^{0}-\theta_{i}^{0}\right)$, one can write

$$
\alpha \Omega_{i}-\alpha \omega_{c}+\sigma \sum_{j=1}^{N} A_{i j} \sin \left(\theta_{j}^{0}-\theta_{i}^{0}\right)=0 .
$$

If we rename $\tilde{\omega}_{i}=\alpha\left(\Omega_{i}-\omega_{c}\right)$, the previous equation reads

$$
\sigma \sum_{j=1}^{N} A_{i j} \sin \left(\theta_{j}^{0}-\theta_{i}^{0}\right)=-\tilde{\omega}_{i}
$$

which turns out to be fundamental in order to determine the values of initial phases $\theta_{i}^{0}$ necessary to obtain a frequency-synchronized solution.

In order to determine the linear stability, consider the evolution of system (4) subject to small perturbations around the desired (frequency-synchronized) solution, i.e., $\theta_{i}=\theta_{i}^{t}+\delta \theta_{i}$, $\omega_{i}=\omega_{c}+\delta \omega_{i}$. Using the equality $\cos \left(\theta_{i}^{t}-\theta_{j}^{t}\right)=\cos \left(\theta_{i}^{0}-\theta_{i}^{0}\right)$ and the fact that $\dot{\omega}_{c}=0$ and $\dot{\Omega}_{i}=0$, we obtain

$$
\begin{aligned}
\delta \dot{\theta}_{i} & =\delta \omega_{i}, \\
\delta \dot{\omega}_{i} & =-\alpha \delta \omega_{i}+\sigma \sum_{j=1}^{N} A_{i j} \cos \left(\theta_{j}^{0}-\theta_{i}^{0}\right)\left(\delta \theta_{j}-\delta \theta_{i}\right) .
\end{aligned}
$$

The system of $2 \mathrm{~N}$ ordinary differential equations can be rewritten in a block matrix form

$$
\left(\begin{array}{l}
\delta \dot{\theta} \\
\delta \dot{\omega}
\end{array}\right)=\left(\begin{array}{cc}
\mathbf{0} & \mathbb{I} \\
\sigma \mathbf{M} & -\alpha \mathbb{I}
\end{array}\right)\left(\begin{array}{l}
\delta \theta \\
\delta \omega
\end{array}\right)
$$

where $\delta \theta \equiv\left(\delta \theta_{1}, \ldots, \delta \theta_{N}\right)^{T}, \delta \omega \equiv\left(\delta \omega_{1}, \ldots, \delta \omega_{N}\right)^{T}, \mathbb{I}$ is the $N \times N$ unit matrix, and $\mathbf{M}$ represents the $N \times N$ Laplacian matrix of a weighted undirected graph

$$
\mathbf{M}=\left(\begin{array}{ccc}
-\sum_{j} A_{1 j} \cos \left(\theta_{j}^{0}-\theta_{1}^{0}\right) & \cdots & A_{1 N} \cos \left(\theta_{1}^{0}-\theta_{N}^{0}\right) \\
\cdots & \cdots & \cdots \\
A_{N 1} \cos \left(\theta_{N}^{0}-\theta_{1}^{0}\right) & \cdots & -\sum_{j} A_{N j} \cos \left(\theta_{j}^{0}-\theta_{N}^{0}\right)
\end{array}\right) .
$$

Note that, although the synchronous state is time-dependent, linearization around this state gives a Jacobian matrix with constant 
coefficients. Hence, the stability of the frequency-synchronized solution can be analyzed by solving the eigenvalue problem of the $2 N \times 2 N$ Jacobian matrix appearing in Eq. (13),

$$
\operatorname{det}(\mathbf{G}-\lambda \mathbb{I}) \equiv\left|\begin{array}{cc}
-\lambda \mathbb{I} & \mathbb{I} \\
\sigma \mathbf{M} & (-\lambda-\alpha) \mathbb{I}
\end{array}\right| .
$$

By using Schur's complement, we obtain $|\mathbf{G}|=\left|G_{11}\right|\left|\frac{\mathbf{G}}{G_{11}}\right|=\left|G_{11}\right|$ $\left|G_{22}-G_{21} G_{11}{ }^{-1} G_{12}\right|$. Thus, we are able to derive an expression for $\operatorname{det}(\mathbf{G}-\lambda \mathbb{I})=|-\lambda \mathbb{I}|\left|(-\lambda-\alpha) \mathbb{I}-\sigma \mathbf{M}(-\lambda \mathbb{I})^{-1} \mathbb{I}\right|=-\lambda^{N}$ $\left|(-\lambda-\alpha) \mathbb{I}+\lambda^{-1} \sigma \mathbf{M}\right|$ and finally obtain

$$
\left|\left(\lambda^{2}+\lambda \alpha\right) \mathbb{I}-\sigma \mathbf{M}\right|=0
$$

If we denote the eigenvalues of matrix $\mathbf{M}$ by $\mu$, i.e., $|\mu \mathbb{I}-\mathbf{M}|=0$, then we have to solve quadratic equations of the type

$$
\lambda^{2}+\lambda \alpha-\sigma \mu=0
$$

in order to determine the eigenvalues of matrix $\mathbf{G}$ defined in Eq. (14). The eigenvalues are given by

$$
\lambda=\frac{-\alpha \pm \sqrt{\alpha^{2}+4 \mu \sigma}}{2}
$$

and depending on the properties of $\mathbf{M}$ the following holds:

(i) $\exists \mu>0 \Leftrightarrow \exists \lambda>0$.

(ii) If matrix $\mathbf{M}$ is stable, then $\mathbf{G}$ is also stable.

(iii) If $\cos \left(\theta_{j}^{0}-\theta_{i}^{0}\right)>0\left(\left|\theta_{j}^{0}-\theta_{i}^{0}\right|<\frac{\pi}{2}\right)$, then $\mathbf{M}$ is a diagonally dominant matrix. This means $\left|M_{i i}\right| \geq \sum_{j \neq i}\left|M_{i j}\right|$.

Thus, we can conclude that it is not possible to find an unstable solution in the neighborhood of $\left\{\theta_{i}^{0}\right\}=0$. Furthermore, we know that any symmetric dominant matrix $\mathbf{R}$ is positive definite if $R_{i i}>0$, thus $\lambda(\mathbf{R}) \geq 0$. In our case, matrix $-\mathbf{M}$ satisfies this conditions, thus $\mu \leq 0$. In accordance with (ii), it comes straightforward that the whole system (13) is stable with respect to small perturbations. These results are in accordance with previous stability analyses performed in Kuramoto systems with inertia. ${ }^{25,39-41}$

\section{NUMERICAL SOLUTION OF EIGENVALUE PROBLEM}

In this section, we explicitly solve the eigenvalue problem stated in Sec. V. In order to perform the stability analysis of a frequencysynchronized solution, we need to find the phases which satisfy the condition expressed in Eq. (11). This means solving an $N$ dimensional system with $N$ unknown variables, namely, the phases $\theta_{i}$. This system may have several solutions, the number of which depends on the system parameters. For instance, low values of the coupling strength $K$ might not admit any solution at all. Nevertheless, for a proper parameter choice, there exist phases $\theta_{i}^{*}$ satisfying Eq. (11), thus describing trajectories a frequency-synchronized system follows. Once the set of phases $\theta_{i}^{*}$ is found, we will insert them into Eq. (13) to solve the eigenvalue problem of the Laplacian matrix M. This, finally, will enable us to characterize the stability of the frequency-synchronized solution.
We are looking for a set of initial phases $\theta_{i}^{*}$ such that the following equation holds:

$$
\mathrm{F}_{i}\left(\theta^{*}\right)=\tilde{\omega}_{i}+\sigma \sum_{j=1}^{N} A_{i j} \sin \left(\theta_{j}^{*}-\theta_{i}^{*}\right)=0
$$

This multidimensional problem can be solved numerically by using the Levenberg-Marquardt algorithm, ${ }^{44}$ which represents a combination of a Gauss-Newton algorithm and the method of gradient descent. The algorithm minimizes, with an iterative procedure, the sum $\sum\left|F_{i}\right|^{2}$, given an initial guess for $\theta_{i}^{0}$. After a certain number of iterations, the algorithm converges to a local minimum near the initial guess for the phases $\theta_{i}^{0}$. However, in order to guarantee that the obtained solution is a true solution for the system of Eq. (17), it should fulfill the condition $\sum\left|F_{i}\right|^{2} \equiv 0$. The trivial choice for the initial conditions $\left\{\theta_{i}^{0}\right\} \approx 0$ gives rise to a suitable solution only if the coupling $K$ is sufficiently strong. A heuristic explanation for this is that, in the limit $K \rightarrow \infty$, the choice of phases $\theta_{i}^{*} \equiv 0$ is always the solution, independent of $\tilde{\omega}_{i}$ and coupling matrix $\mathbf{A}$. On the other hand, it is always possible, especially for large $K$, to find solutions which are quite distinct from the solution obtained at initial zero phases. Recalling that $\left|\theta_{j}^{0}-\theta_{i}^{0}\right|<\frac{\pi}{2}$ must be satisfied in order to ensure that matrix $\mathbf{M}$ is diagonally dominant, these new solutions will be unstable. Finally, once a set of phases $\theta_{i}^{*}$ which minimizes the function is found, it is possible to solve the eigenvalue problem for the matrix $\mathbf{M}$. The eigenvalue with maximum real part determines the stability of the state, while $\mu=0$, which is always present in the Laplacian corresponding to the invariance of the model under uniform phase shift, will not be considered in the following.

Our system is characterized by a sparse network of Kuramoto oscillators with inertia with a symmetric bimodal frequency distribution, whose parameters $m=6, p=0.20$, and $N=500$ are the same as in Fig. 1. By means of the Levenberg-Marquardt algorithm, we are able to obtain a set of phases $\theta_{i}^{*}$, which fulfills the condition for frequency synchronization equation (17). However, as previously discussed, it is not always possible to find a solution (stable or unstable) to our system for arbitrary $K$; therefore, we estimate the critical coupling $K_{c}$, below which the nonlinear system (17) has no solutions. Without loss of generality, we choose as initial guess for the phases $\theta_{i}^{0} \equiv 0$ which is close to the true phases solving Eq. (17). The results are illustrated in Fig. 2. In particular, we can observe that no solution can be found for $K<K_{c}=5.8$ while we can always find a solution for $K>K_{c}$. If $K$ is only slightly above the critical value $K_{c}$, then few solutions are admittable for phases $\theta_{i}^{*}$. For $K$ slightly above $K_{c}$, the coupling is not sufficiently strong to suppress the phase differences $\left|\theta_{j}^{0}-\theta_{i}^{0}\right|$, and for this reason, the global order parameter of $r \approx 0.9$. The corresponding maximum Lyapunov exponent (disregarding $\lambda=0$ ) is negative. By further increasing $K$, a stable solution is obtained for smaller phase differences $\left|\theta_{j}^{0}-\theta_{i}^{0}\right|$, which contribute to an increase of the global order parameter $r$. The corresponding maximum eigenvalue $\mu_{\max }$ of $\mathbf{M}$ decreases accordingly (inset of Fig. 2). The eigenvalue $\lambda$ determining the stability of the frequencysynchronized solution is given by Eq. (15) and has the real part $\operatorname{Re}(\lambda)=-\alpha / 2=-0.083$ for $4|\mu| \sigma>\alpha^{2}$ which holds for all $K>$ 


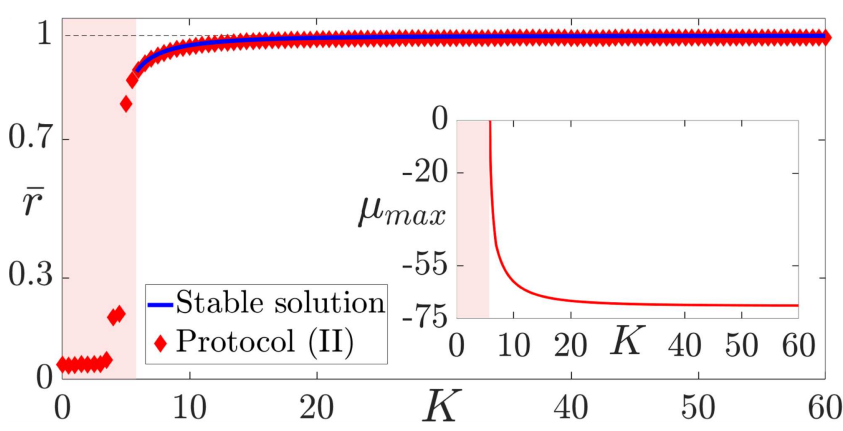

FIG. 2. Average global order parameter $\bar{r}$ as a function of coupling strength $K$. The shaded red area indicates the region in which no frequency-synchronized solutions can be found. The blue solid line refers to the stable solution, whereas the sequence of simulations obtained by running protocol (II) is denoted by red diamonds. Inset: maximum eigenvalue $\mu_{\max }$ as a function of coupling strength $K$. Other parameters as in Fig. 1.

$K_{c}=5.8$ where the frequency-synchronized solution exists. Similar results have been found for a network with asymmetric bimodal frequency distribution (see the Appendix).

It is remarkable that the stable solution obtained by iterating the algorithm with initial phases set to zero coincides with the simulations obtained by performing protocol (II) (at least outside the shaded area). This suggests that the hysteretic loop observed in Fig. 1, due to the presence of the inertial term, strongly depends on the initial conditions for the phases. It follows that, by choosing an appropriate set of initial phases $\theta_{i}^{0}$, the power grid can reach synchronization faster without passing through intermediate states (i.e., traveling and standing waves) as in protocol (I).

The solution of the eigenvalue problem, related to a specific set of phases $\theta_{i}^{*}$, gives rise to both stable and unstable solutions. An example of this is illustrated in Fig. 3, where stable (unstable) solutions are reported in panels a and $b$ (c and $d)$. In particular, panel (a) depicts the spatiotemporal evolution of the phases $\theta_{i}$ for a stable solution $\left[\operatorname{Re}\left(\lambda_{\max }\right)=-0.083\right]$ : the initial set of phases $\theta_{i}^{*}$ is approximately equal to zero while, as time changes, $\theta_{i}$ change at equal rates for all the nodes, thus indicating that they move with the same constant phase velocity $\omega_{c}$, as confirmed by the evenly distributed greenish color in panel (b). Since the natural frequencies $\Omega_{i}$ are randomly distributed according to a bimodal Gaussian distribution with opposite means $\Omega_{0}= \pm 2$, the arithmetic mean $\omega_{c}$ given in Eq. (9) is close to 0 .

A different scenario arises for the unstable solution, see the bottom panels of Fig. 3. Initially, all the phases $\theta_{i}^{*}$ obtained by solving Eq. (17) are uniformly distributed on a limit cycle. When the system starts evolving, the phases $\theta_{i}$ evolve nontrivially in time and they change at equal rates up to the time of $t \approx 9$. Afterwards, the system starts oscillating until a new solution is reached. A confirmation of this behavior can be found by analyzing the temporal evolution of the corresponding frequencies $\omega_{i}$ shown in panel (d). Here, we see that the frequencies lose their constant value at $t \approx 9$, corresponding to frequency synchronization death. For different sets of initial conditions, while keeping the same coupling constant $K$, it is also possible to observe cases where the system leaves the frequency-synchronized
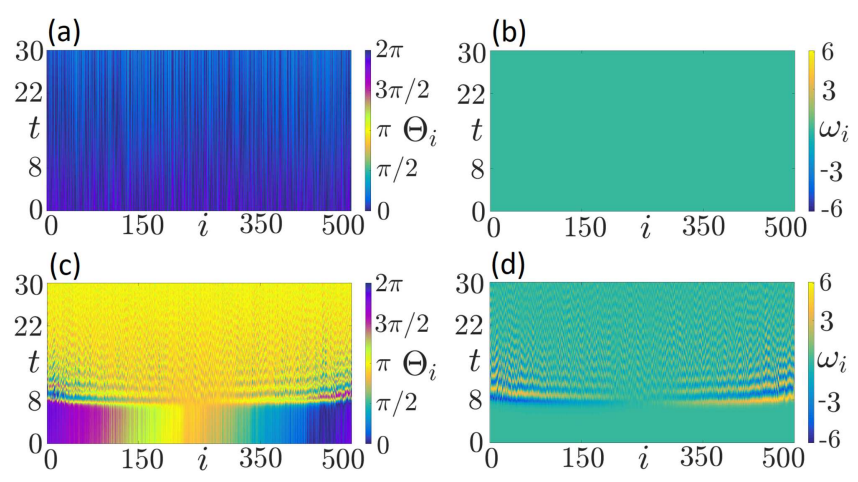

FIG. 3. Spatiotemporal evolution of phases $\theta_{i}$ and frequencies $\omega_{i}$, which satisfy the condition for frequency-synchronized solution. Stable solution: (a) phases; (b) frequencies; parameters: $\operatorname{Re}\left(\lambda_{\max }\right)=-0.083, K=10$. Unstable solution: (c) phases; (d) frequencies; parameters: $\lambda_{\max }=2.41, K=70$. Other parameters: as in Fig. 1.

solution even earlier with larger $\operatorname{Re}\left(\lambda_{\max }\right)$. Finally, for increasing time $t$, the system approaches another stable state.

\section{CONTROL OF UNSTABLE STATES}

In this section, we aim to control the stability of the solution satisfying Eq. (17). In particular, we want to stabilize frequencysynchronized solutions obtained for sets of initial phases which violate the condition for the diagonal dominance of matrix $\mathbf{M}$, i.e., for sets of phases whose differences $\left|\theta_{j}^{0}-\theta_{i}^{0}\right| \geq \frac{\pi}{2}$. For this purpose, we introduce a control term $u_{i}$ into the original system (4),

$$
\begin{aligned}
& \dot{\theta}_{i}=\omega_{i}, \\
& \dot{\omega}_{i}=\alpha \Omega_{i}-\alpha \omega_{i}+\sigma \sum_{j=1}^{N} A_{i j} \sin \left(\theta_{j}-\theta_{i}\right)+u_{i} .
\end{aligned}
$$

A linear control technique can be applied to the nonlinear system since the linearization around the synchronous state leads to a time-independent Jacobian matrix and hence to an eigenvalue problem of the Jacobian equivalent to that of an unstable equilibrium. By definition, the stability of equilibrium points is determined by the eigenvalues of the Jacobian, which corresponds to the matrix of the linearized system. The linearized equation (13) will change accordingly

$$
\left(\begin{array}{l}
\delta \dot{\theta} \\
\delta \dot{\omega}
\end{array}\right)=\mathbf{G}\left(\begin{array}{l}
\delta \theta \\
\delta \omega
\end{array}\right)+\mathbf{B} u,
$$

where $\mathbf{G} \in R^{2 N \times 2 N}$ and $\mathbf{B}=\left(\begin{array}{c}\mathbb{O}_{N} \\ \mathbb{I}_{N}\end{array}\right) \in R^{2 N \times N}$.

In particular, the control term $u$ can be chosen as a feedback control loop such that

$$
u=-\mathbf{C}\left(\begin{array}{l}
\delta \theta \\
\delta \omega
\end{array}\right)
$$



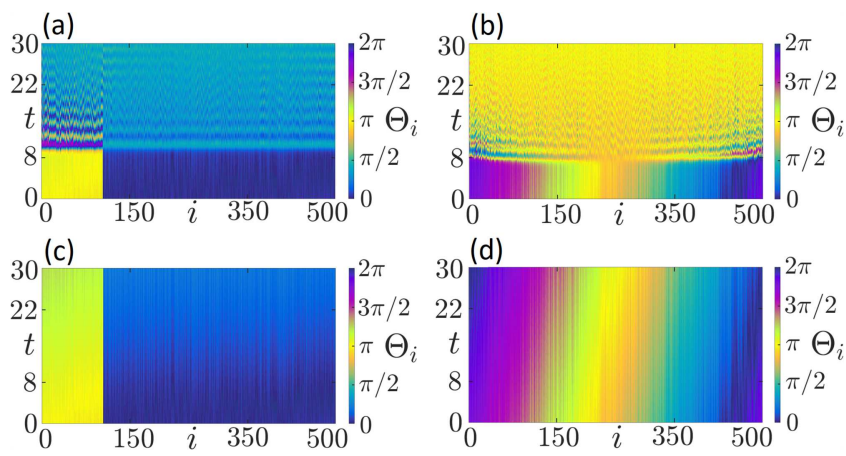

FIG. 4. Spatiotemporal evolution of phases $\theta_{i}$ without (top panel) and with control (bottom panel) for a symmetric bimodal frequency distribution. Left column: $K=50$ and initial phases $\theta_{1}^{*}=\cdots=\theta_{150}^{*}=\pi, \theta_{151}^{*}=\cdots=\theta_{500}^{*}=0,(\mathrm{a})$ control off, $\lambda_{\max }=2.802$; (c) control on, $\lambda_{\text {ctrl }}=-0.759$. Right column: $K=70$ and uniformly distributed initial phases, (b) control off, $\lambda_{\max }=2.41$; (d) control on, $\lambda_{\text {ctrl }}=-0.823$. Other parameters: as in Fig. 1 .

where $\mathbf{C} \in R^{N \times 2 N}$ is chosen to minimize the following cost functional:

$$
J(u)=\int_{0}^{\infty}\left\|\left(\begin{array}{c}
\delta \theta(t) \\
\delta \omega(t)
\end{array}\right)\right\|^{2}+\|u(t)\|^{2} d t .
$$

This problem is solved via the application of a linear quadratic regulator for each set of phases $\theta_{i}^{*}$. Basically, the regulator chooses the time-independent matrix $\mathbf{C}$ such that the eigenvalues for the closedloop system $\lambda_{\text {ctrl }}$ are nonpositive when solving the eigenvalue problem for matrix $\mathbf{G}-\mathbf{B C}$. Thus, the frequency-synchronized solution obtained from Eq. (17) is stabilized for each particular set of chosen phases $\theta_{i}^{*}$, and, regardless of the initial phase differences $\left|\theta_{j}^{0}-\theta_{i}^{0}\right|$, we are always able to obtain a stable solution if $K>K_{c}$.

The efficiency of the control method is shown in Fig. 4 for two different sets of initial phases $\theta_{i}^{*}$. In particular, in panel (a), we use an initial set of phases which consists of approximately $70 \%$ of phases set to $\theta_{0}^{*}=0$ and the remaining $30 \%$ set to $\theta_{0}^{*}=\pi$, which does not fulfill the criterion for the diagonal dominance of matrix $\mathbf{M}$, since $\left|\theta_{j}^{0}-\theta_{i}^{0}\right|>\frac{\pi}{2}$. The system remains in this frequency-synchronized state until the instability triggers oscillations at $t \approx 10$. At this point, the system leaves the unstable frequency-synchronized solution and the phases change at different rates, thus indicating that frequency synchronization is lost until another attractor is reached. The corresponding bottom panel (c) illustrates the temporal evolution of the system, for the same initial set of phases, when control is implemented and turned on. In this case, the system does not leave the initial state for sufficiently large time, even though the initial set of phases does not fulfill the condition of the diagonal dominance of matrix $\mathbf{M}$. The system turns out to be stabilized by the action of control. The same behavior is observed for an initial set of uniformly distributed phases, see panel (b). After the application of control, we obtain a stable solution which is reminiscent of a traveling wave. Note that, for both cases (b) and (d), the corresponding space-time plots with respect to the frequency would look exactly as in Fig. 3(b).
As a proof of principle, here we have applied the control method to a diluted Erdös-Renyi network with symmetric bimodal frequency distribution. In the Appendix, we report a few more examples where the control method turns out to work, i.e., a diluted Erdös-Renyi network with asymmetric bimodal frequency distribution, and a realistic power grid (Italy) with bimodal $\delta$-distribution of frequencies.

\section{INTERPLAY OF NOISE AND DISORDER}

Finally, we investigate the influence of temporal power fluctuations of generators. Strong temporal fluctuations are a signature of renewable energy-based power grids, e.g., wind turbines and photovoltaics. For this purpose, we add Gaussian white noise to the system considered so far

$$
m \ddot{\theta}_{i}+\dot{\theta}_{i}=\Omega_{i}+\frac{K}{N_{c}} \sum_{j=1}^{N} A_{i j} \sin \left(\theta_{j}-\theta_{i}\right)+\sqrt{2 D} \xi_{i}(t),
$$

where $\xi_{i}$ denotes Gaussian white noise defined by $\left\langle\xi_{i}\right\rangle=0$ and $\left\langle\xi_{i}(t) \xi_{j}(s)\right\rangle=\delta_{i j} \delta(t-s)$ and $D$ is the noise intensity.

Networks of Kuramoto oscillators with inertia subject to white noise have been recently investigated in Refs. 21,28 , and 46 to mimic stochastic power fluctuations and to compute the synchronization transition scenarios. In particular, Tumash et al. ${ }^{28}$ have investigated the influence of noise on the synchronization transition for globally coupled networks; here, we extend these previous studies to sparse networks.

In order to find out how the external noise changes the properties of a diluted network, we first investigate the synchronization transition for a random network with average connectivity $N_{c}=0.1 N$ (i.e., dilution parameter $p=0.10$ and $90 \%$ of links removed) under the impact of Gaussian white noise of different intensities. The distribution of natural frequencies is chosen to be a symmetric bimodal Gaussian. In particular, Fig. 5(a) shows the results for $\sqrt{2 D}=5$, while in Fig. 6(a), a noise of intensity $\sqrt{2 D}=15$ is considered. As expected, the hysteretic region, identified by the vertical dotted green lines at $K_{1}^{c}=25$ and $K_{2}^{c}=5$ for $\sqrt{2 D}=5$, becomes smaller in comparison to what we observe in Fig. 1(a). Moreover, the system reveals a smaller K-interval where travelling waves occur in the upsweep. As the noise is increased, standing waves begin to disappear as well and intermediate states are not detected any more. Figure 6(a) confirms this: for higher noise intensity, the hysteretic region shrinks considerably and the intermediate states, like travelling and standing waves, which were still present for lower noise, are no more accessible. Therefore, while, in Fig. 5(a), both noise and dilution seem to play a minimal role, in Fig. 6(a), we observe the typical effect of noise at high intensity: the system reaches the synchronized state for smaller coupling values and without exploring intermediate states. This is consistent with the results found in Ref. 28, where it was suggested that disorder and noise have an equivalent effect, both aimed at reducing the hysteretic region and preventing the system from reaching intermediate states (i.e., travelling and standing waves). Panels (b)-(d) of Fig. 5 illustrate the features of the frequency-synchronized state appearing at $K=30$ with $\bar{r}=0.96$. Panel (b) shows that almost all frequencies are synchronized, while phases and instantaneous phase velocities are not strictly correlated due to the effect of noise, see panels (c) and (d). Moreover, both noise 

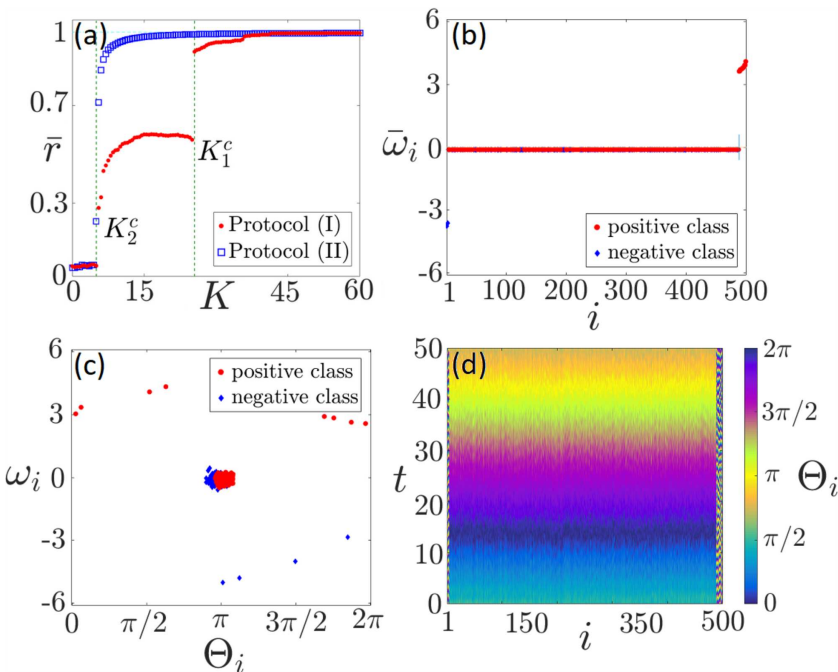

FIG. 5. (a) Time-averaged global order parameter $\bar{r}$ as a function of coupling constant $K$ for a randomly coupled network with a dilution parameter of $p=0.10$ and stochastic dynamics with noise intensity $\sqrt{2 D}=5$, obtained by following protocol (I) (upsweep, red filled circles) and protocol (II) (downsweep, blue empty squares). The vertical dotted green lines denote the boundaries of the hysteretic region. $K_{1}^{c}=25$ and $K_{2}^{c}=4.5$. (b) Average phase velocity $\bar{\omega}_{i}$ for $K=30$ (frequency-synchronized solution). The elements related to the positive (negative) distribution of natural frequencies are denoted by red circles (blue diamonds). (c) Instantaneous phase velocity $\omega_{i}$ vs phase $\theta_{i}$ for all $i \in(1, \ldots, 500)$ (snapshot). (d) Spatiotemporal evolution of phases $\theta_{i}$. For all panels, a centered, symmetric bimodal distribution of the natural frequencies has been used. Other parameters as in Fig. 1.

and dilution (spatial disorder) contribute to decreasing the value of coupling strength $K$ at which partial frequency synchronization is reached $\left(K_{1}^{c}=25\right)$, as compared to the all-to-all coupled case without noise $\left(K_{P S}=31\right)$. For higher noise intensity, the network is already fully frequency-synchronized at $K=30$, even though it is not phase synchronized [see Fig. 6, panels (b)-(d)].

As a second setup, we consider a random network with average connectivity $N_{c}=0.1 \mathrm{~N}(p=0.1)$, where the distribution of natural frequencies is chosen to be a noncentered, asymmetric bimodal Gaussian distribution in order to mimic the presence of $20 \%$ of generators and $80 \%$ of loads in the network. When asymmetry is introduced into the network, it becomes more difficult to reach full synchronization. It is achieved for $K>52$ in the case of low noise intensity and for $K>40$ in the case of high noise intensity [see Figs. 7(a) and 8(a), respectively]. However, it turns out that partial synchronization can be reached for lower $K$ values as compared to the symmetric case, since $K_{1}^{c}$ is always smaller than in the previous setup, irrespective of the noise level. The effects of noise and dilution are visible even in this case, since for higher noise intensity, we observe limited accessibility to intermediate states. As a result, the system jumps from the asynchronous state directly to a partially synchronized state with high coherence. Note that full frequency synchronization is achievable in the upsweep for lower $K$ values when noise is increased [Fig. 8(a)]. A comparative analysis with respect
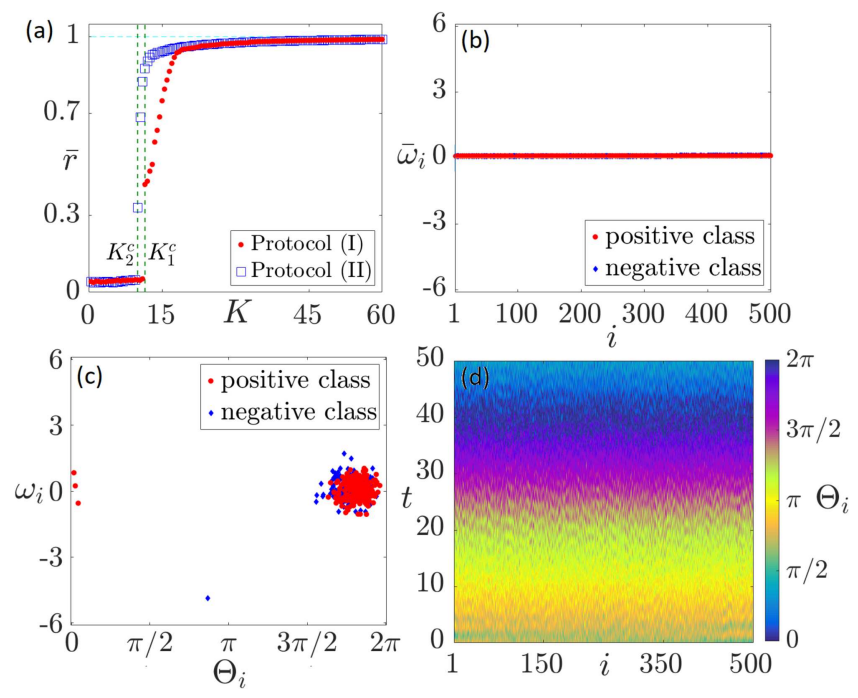

FIG. 6. Same as Fig. 5 for a randomly coupled network with stochastic dynamics and noise intensity $\sqrt{2 D}=15 . K_{1}^{c}=11.5$ and $K_{2}^{c}=10.0$.

to the one depicted in Figs. 5 and 6 is shown in Figs. 7, 8 [panels (b)-(d)] for $K=30$. In the diluted network with asymmetric frequency distribution and low noise intensity (Fig. 7), we observe a two-frequency cluster state for the chosen coupling value: clusters are reminiscent of the original separation (i.e., generators synchronized at $\bar{\omega}_{i}=\Omega_{0^{+}}=3.2$ and loads clustered at $\bar{\omega}_{i}=-\Omega_{0^{-}}=-0.8$ at $K=0$ ), where some generators (red) become synchronized to the loads (blue) at an average frequency $\approx-0.2$ close to that of the loads, and the coupling is not yet sufficient to merge the two clusters into a single one. For high noise intensity (Fig. 8), note that the cluster structure is no longer present at $K=30$, but it has given a way to frequency synchronization, except for a few outliers: as in the previous case, the transition to synchronization takes place for lower coupling values when the noise is increased.

Finally, numerical results are presented for the Italian highvoltage power grid for low noise intensity $\sqrt{2 D}=5$ (Fig. 9) and for high noise intensity $\sqrt{2 D}=15$ (Fig. 10). This network is characterized by a quite low average connectivity $\left\langle N_{c}\right\rangle=2.865$, due to the geographical distributions of the nodes along Italy; therefore, the synchronization transition exhibits no hysteresis and has become continuous, as shown in Ref. 24. When the transition is continuous, the noise does not play a constructive role any more and just smears out over a larger interval of $K$ the transition to synchronization that takes place for higher $K$ values, as compared to the deterministic case. While in the deterministic case $K_{1}^{c}=26$ holds, for low noise intensity, $K_{1}^{c}=35$ and, for high noise intensity, $K_{1}^{c}=30$ is found. However, the Kuramoto order parameter, defined in Eq. (3), characterizes phase synchronization rather than frequency synchronization, and we know that in our system frequency synchronization does not imply phase synchronization. In Fig. 9(b), we see that the average frequency is zero, already at $K=30$, which corresponds to a frequencysynchronized regime. In panel (c), the instantaneous frequencies are 

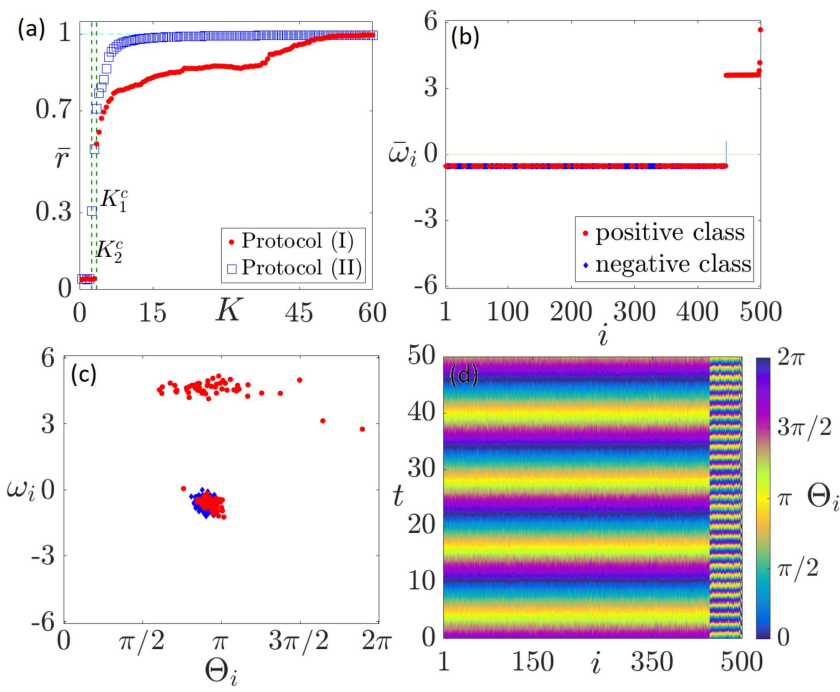

FIG. 7. Same as Fig. 5 for a noncentered, asymmetric bimodal distribution of the natural frequencies modelling a random network made of $20 \%$ of generators and $80 \%$ of consumers $\left(p_{g}=0.2, \Omega_{0^{+}}=3.2, \Omega_{0^{-}}=0.8\right)$, and stochastic dynamics with noise intensity $\sqrt{2 D}=5 . K_{1}^{c}=3.5$ and $K_{2}^{c}=2.5$.

shown to be localized around zero, only slightly smeared out due to the action of noise, while the distribution of phases is widely spread over all possible values. The randomly spread-out phases show up as a salt-and-pepper pattern in the space-time plot in panel (d) but by renumbering of the nodes according to increasing phase
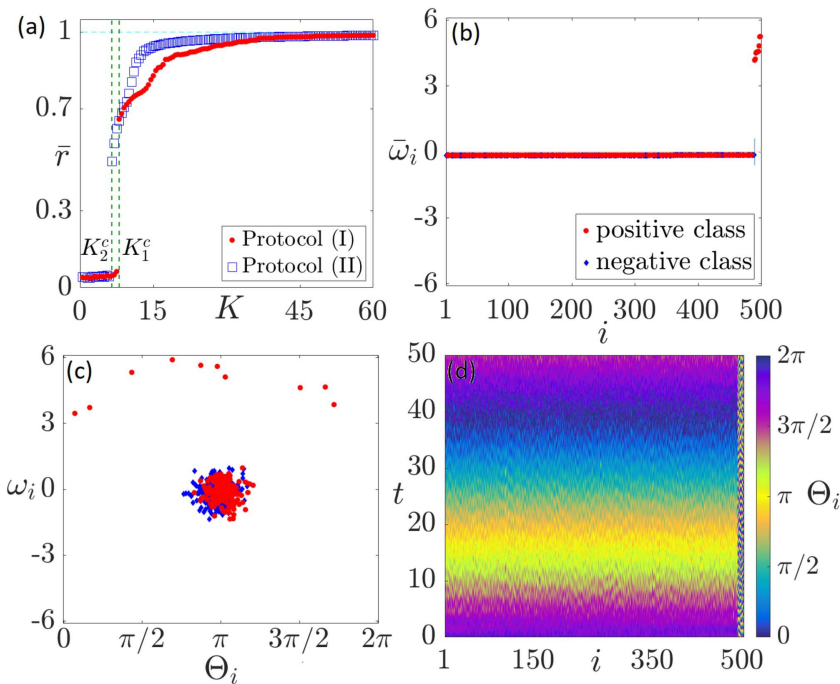

FIG. 8. Same as Fig. 5 for a noncentered, asymmetric bimodal distribution of the natural frequencies modelling a random network made of $20 \%$ of generators and $80 \%$ of consumers $\left(p_{g}=0.2, \Omega_{0^{+}}=3.2, \Omega_{0^{-}}=0.8\right)$, and stochastic dynamics with noise intensity $\sqrt{2 D}=15 . K_{1}^{c}=8.0$ and $K_{2}^{c}=6.5$.
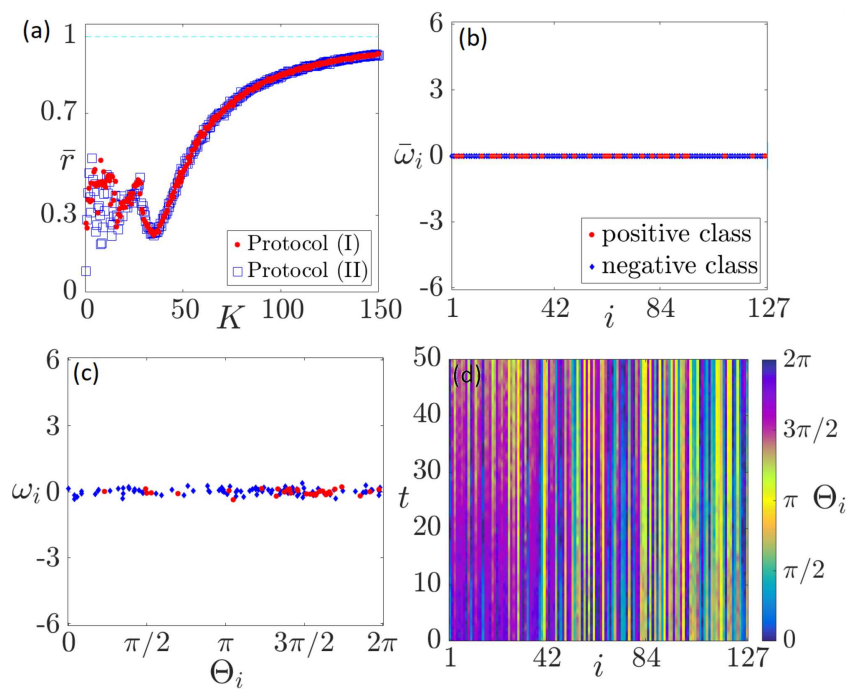

FIG. 9. Same as Fig. 5 for the Italian high-voltage grid and stochastic dynamics with noise intensity $\sqrt{2 D}=5$.

the regular phase-locking space-time pattern might be made better visible. For high noise intensity, frequency synchronization cannot be achieved any more at $K=30$ [see Figs. 10(a)-10(c)], and the network splits into two parts corresponding to a 2 -frequency cluster, where the phases are more synchronized in the left-hand part $i=1, \ldots, 50$ (North Italy), compared to the right-hand part $i=$ $51-127$ (South Italy). Therefore, we can conclude that, for highly sparse networks, the Kuramoto order parameter is not an appropriate
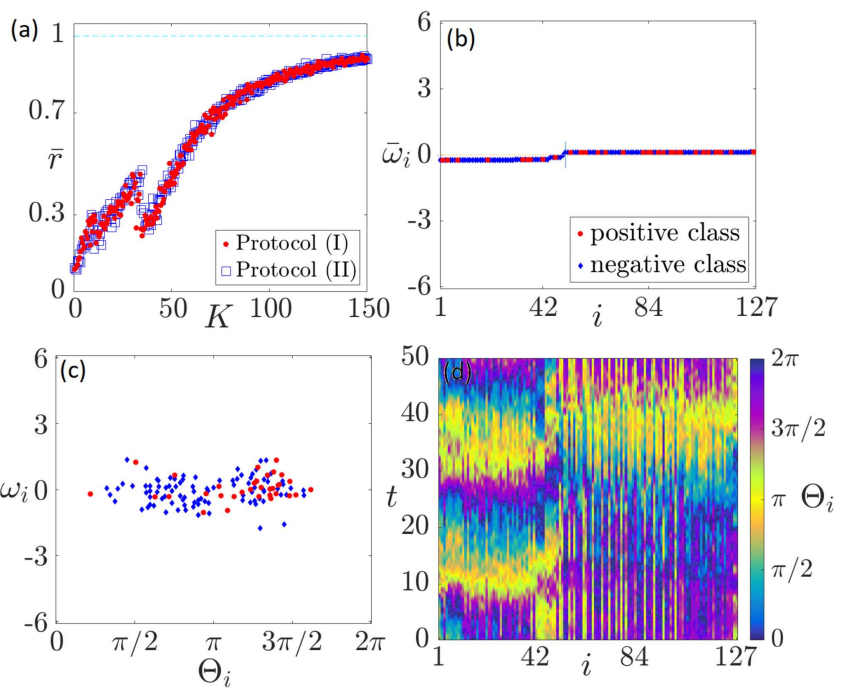

FIG. 10. Same as Fig. 5 for the Italian high-voltage grid and stochastic dynamics with noise intensity $\sqrt{2 D}=15$. 

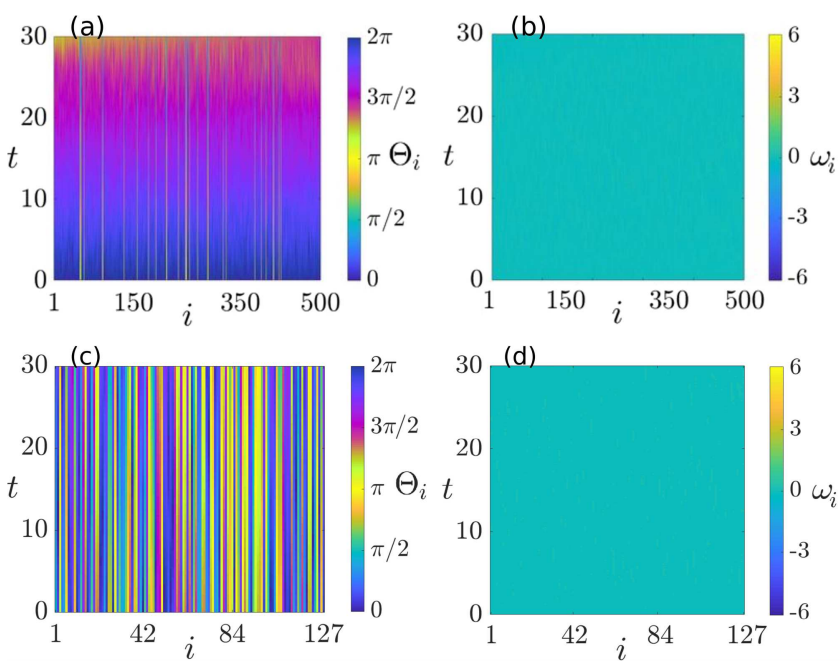

FIG. 11. Upper panels: Spatiotemporal evolution of phases $\theta_{i}(a)$ and frequencies $\omega_{i}$ (b) with control for a randomly coupled network with dilution parameter $p=0.10$ and stochastic dynamics with noise intensity $\sqrt{2 D}=5$. Nodes are distributed according to the following percentage: $20 \%$ generators and $80 \%$ loads $\left(p_{g}=0.2, \Omega_{0^{+}}=3.2, \Omega_{0^{-}}=0.8\right) . K=70$. Other parameters as in Fig. 7 . Lower panels: spatiotemporal evolution of phases $\theta_{i}$ (c) and frequencies $\omega_{i}$ (c) with control for the Italian high-voltage power grid with noise intensity $\sqrt{2 D}=5$ at $K=70$. Other parameters as in Fig. 9 .

measure to characterize the (frequency) synchronization level of the network, as noted previously, ${ }^{29,47}$ and the noise renders frequency synchronization more difficult to be attained, since it spreads the instantaneous frequencies around the mean synchronization value.

\section{CONTROL OF UNSTABLE NOISY STATES}

This section is devoted to the application of the control method to sparse networks with stochastic power fluctuations. Based upon the characterization of synchronization in sparse networks of Kuramoto oscillators with inertia under the influence of noise in Sec. VIII, we will now investigate the efficiency of control, which was studied for deterministic dynamics in Sec. VII, in the following more realistic setups with noise: (i) a randomly coupled network with asymmetric bimodal frequency distribution and (ii) Italian high-voltage power grid. The results are shown in Fig. 11. The control method is able to stabilize unstable solutions and to secure a frequency-synchronized state for low noise intensity (e.g., $\sqrt{2 D}=5$ ), while it is not able to succeed if the noise intensity is too high (results not shown). Note that even in the presence of control, frequency synchronization does not imply phase synchronization. In particular, for the Italian grid [panel (c)], the salt-and-pepper pattern of the phases in the space-time plot remains analogous to the one shown in Fig. 9(d) for a lower coupling value and no control. For the random network with asymmetric bimodal frequency distribution, some solitary states with different phase remain [Fig. 11(c)].

\section{CONCLUSIONS}

In this paper, we have considered sparse networks of Kuramoto oscillators with inertia to investigate the optimal conditions for the emergence of synchronization in power grids. In the center of our study is the theoretical analysis of the stability of the frequencysynchronized state, which is necessary for the stable operation of power grids, and its control by a linear quadratic regulator. Going beyond previous work, ${ }^{28}$ we have used a linear stability analysis to identify unstable frequency-synchronized states in power grids with sparse connectivity and applied feedback control to stabilize them, then extended our study to include stochastic temporal power fluctuations and discussed the interplay of topological disorder and Gaussian white noise for various model configurations, and finally demonstrated that our control scheme also works well under the influence of noise. Results for synthetic Erdös-Renyi random networks with low average connectivity are compared with those obtained by considering a real power grid topology, namely, the grid of Italy.

We have implemented sparse synthetic random networks in order to understand the mechanisms underlying the transition to synchronization and to capture the role played by spatial disorder in randomly diluted networks and temporal noise, typical for decentralized renewable energy sources, in altering the nature of this transition. In power grids, where it is of vital importance to maintain stable frequency synchronization, the details of achieving synchronization cannot be neglected: The transition to synchronization for upsweep and downsweep of the coupling strength, i.e., line capacity, is hysteretic and a finite perturbation can bring the system back to an intermediate state, which is different from the synchronized one. For this reason, we have not limited our analysis to the investigation of the properties of the synchronized state, as it is common in the literature but have given a more general framework of the transition to synchronization, characterizing all intermediate states of partial synchronization.

Within this study, we have provided a linear stability analysis of the frequency-synchronized solution and derived the stability criteria, based on the initial phase differences of the oscillators, and have estimated the critical coupling strength $K_{c}$ above which a frequencysynchronized solution is possible in the deterministic system. For sufficiently large coupling, we have also found unstable solutions that are usually characterized by large differences of initial phases. It turns out that our linear feedback control scheme is very efficient in stabilizing unstable frequency-synchronized solutions for arbitrary initial phases, and all $K>K_{c}$, independent of the chosen frequency distribution and the investigated sparse network. In particular, we have simulated highly-diluted Erdös-Renyi networks with symmetric or asymmetric bimodal frequency distributions, where the asymmetric case corresponds to the realistic situation of a grid with fewer but larger generators and many small consumers. In addition to the Erdös-Renyi networks, we have successfully tested the control scheme with a realistic high-voltage power grid, i.e., the Italian grid.

Furthermore, we have investigated diluted networks with stochastic dynamics due to temporally fluctuating power in order to infer the similarities and differences occurring in the transition to synchronization with respect to the deterministic case. We have added a simple noise term, i.e., Gaussian white noise, rather than correlated noise or intermittent noise, in order to gain insight into 
the general role played by noise in power systems. Real power grids are susceptible to noise, independent of the inclusion of renewable, strongly fluctuating energy sources, and here we use the noise term not specifically to model renewable generators, for which it would be more appropriate to introduce non-Gaussian correlated noise to reproduce their fluctuations. ${ }^{15,17}$ Previously, the transition to synchronization has been investigated mainly in deterministic systems ${ }^{24}$ or in globally coupled networks. ${ }^{28}$ On the other hand, when stochastic systems with Gaussian white noise were considered, ${ }^{21,46}$ the focus has not been on the synchronization transition, thus neglecting possible consequences of hysteresis in power systems. In particular, here we have observed that, for synthetic diluted networks (independent of the frequency distribution), intermediate noise intensities might play a constructive role in lowering the critical coupling value required to reach (almost complete) frequency synchronization, since noise suppresses intermediate states and reduces the hysteretic region. On the other hand, for the Italian high-voltage power grid, for which the transition to synchronization is continuous, high noise intensity prevents the system from reaching frequency synchronization. Finally, we have implemented our control scheme in systems with Gaussian white noise. Our simulations have shown that unstable synchronous states can still be stabilized for low to intermediate noise, both in Erdös-Renyi networks with asymmetric bimodal frequency distribution and in the Italian high-voltage power grid, but for too high noise intensity control fails.

Future perspectives of this work might be aimed at a deeper understanding of the applicability of the control scheme within noisy systems, for non-Gaussian noise and realistic topologies.

\section{ACKNOWLEDGMENTS}

We acknowledge support from the Deutsche Forschungsgemeinschaft (DFG) in the framework of the SFB 910 (Projektnummer 163436311).

\section{APPENDIX: CONTROL FOR ASYMMETRIC FREQUENCY DISTRIBUTION AND ITALIAN GRID}

In order to show the broad applicability of the control method implemented in Sec. VII, we consider in this Appendix two further configurations, both in the absence of noise, (i) a sparse network of Kuramoto oscillators with inertia with an asymmetric bimodal frequency distribution, to mimic the presence of $20 \%$ of generators and $80 \%$ of loads in the grid, and (ii) the Italian high-voltage power grid with bimodal $\delta$-distribution of frequencies.

For the network with asymmetric frequency distribution, by means of the Levenberg-Marquardt algorithm, it is possible to obtain a set of phases $\theta_{i}^{*}$ which fulfills the condition of frequency synchronization, i.e., Equation (17). However, no stable solution can be found for $K<K_{c}=6.5$, while we are always able to obtain a stable solution for $K>K_{c}$. The corresponding maximum eigenvalue $\mu_{\max }$ of $\mathbf{M}$ decreases accordingly, when $K>K_{c}$, see Fig. 12. In comparison with the case of a symmetric bimodal frequency distribution considered in Sec. VI (Fig. 2, inset), here $\mu_{\max }<0$ reaches, for large $K$, smaller absolute values, which means that the synchronous solutions are less stable for the asymmetric case than for the symmetric one.

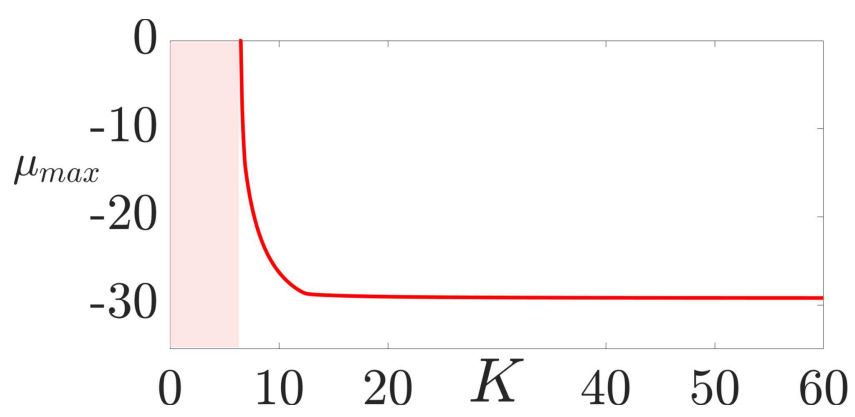

FIG. 12. Maximum eigenvalue $\mu_{\max }$ as a function of coupling strength $K$ for a diluted Erdös-Renyi network with asymmetric bimodal frequency distribution. Nodes are distributed according to the following percentage: $20 \%$ generators and $80 \%$ loads $\left(p_{g}=0.2, \Omega_{0^{+}}=3.2, \Omega_{0^{-}}=0.8\right)$. Other parameters as in Fig. 1.

Then, if we consider an initial set of uniformly distributed phases that does not fulfill the criterion for the diagonal dominance of matrix M, i.e., corresponding to an unstable synchronous state, it turns out that it is always possible to stabilize the system by the action of control (see Fig. 13). In particular, by the action of control, frequency synchronization is achieved together with stability. Note also that most phases are synchronized except for some solitary nodes which remain desynchronized.

When considering the Italian grid, it turns out that the condition for frequency synchronization [Eq. (17)] is well satisfied for all $K>K_{1}^{c}$, but it is not possible to find a set of phases for which
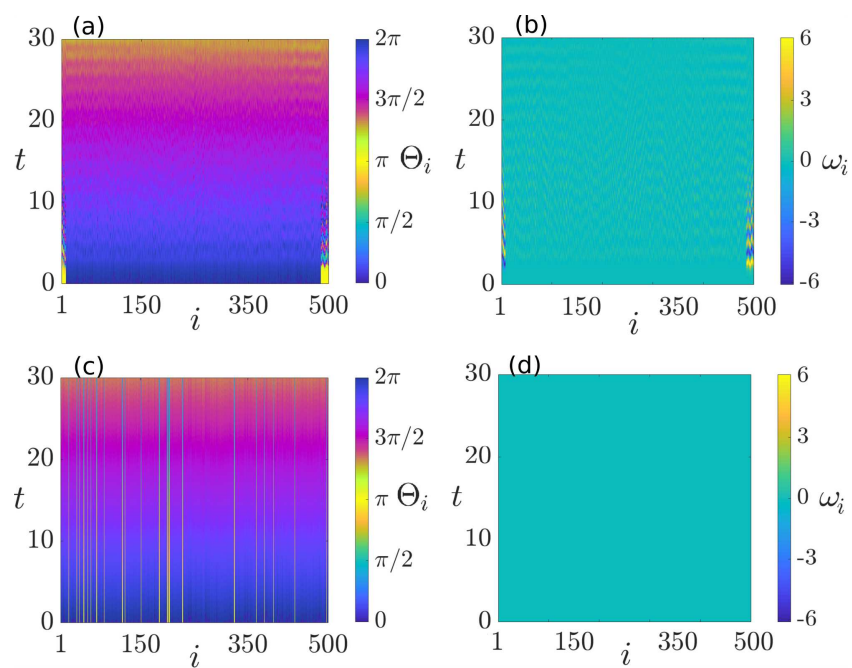

FIG. 13. Spatiotemporal evolution of phases $\theta_{i}$ (left column) and frequencies $\omega_{i}$ (right column) without (top panels) and with control (bottom panels) for an Erdös-Renyi network with asymmetric bimodal frequency distribution. (a) and (b) control off, $\lambda_{\max }=3.622$; (c) and (d) control on, $\lambda_{\text {ctrl }}=-0.733$. Nodes are distributed according to the following percentage: $20 \%$ generators and $80 \%$ loads $\left(p_{g}=0.2, \Omega_{0^{+}}=3.2, \Omega_{0^{-}}=0.8\right) . K=70$. Other parameters as in Fig. 1. 

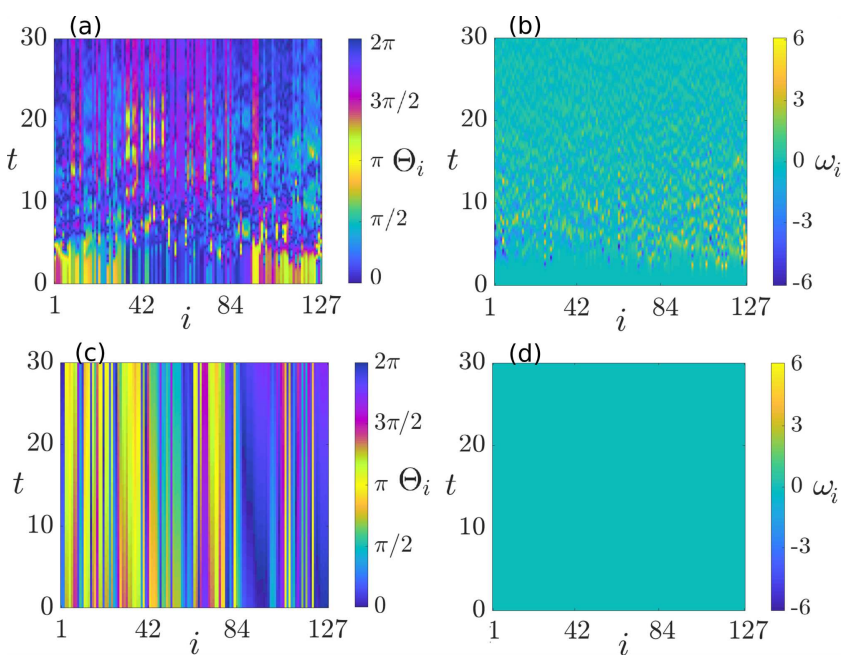

FIG. 14. Spatiotemporal evolution of phases $\theta_{i}$ (left column) and frequencies $\omega_{i}$ (right column) without (top panels) and with control (bottom panels) for the Italian high-voltage power grid. (a) and (b) Control off, $\lambda_{\max }=4.01$; (c) and (d) control on, $\lambda_{\text {ctrl }}=-0.867 . K=70$. Other parameters as in Fig. 9 .

the stability condition $\mu_{\max }<0$ holds. Although we have considered 5000 random sets of initial phases, normally distributed around zero, we have found only unstable solutions when applying the Levenberg-Marquardt algorithm, albeit we cannot exclude that a stable solution still exists for some initial condition. However, what we suspect is that a stable fully synchronized solution does not exist at least for an intermediate range of coupling $(K \leq 27)$ : in this regime, quasiperiodic dynamics arises, ${ }^{24}$ in contrast to the strictly periodic motion that constitutes the solution of Eq. (17). Unstable solutions can be still stabilized via the Linear Quadratic Regulator, as shown in Fig. 14. The control method allows us to obtain a stable frequencysynchronized solution, even though phase synchronization is not achieved but rather a phase-locked state characterized by a Kuramoto order parameter smaller than 1.

\section{REFERENCES}

${ }^{1}$ Intergovernmental Panel on Climate Change, Climate Change 2007: The Physical Science Basis, Agenda 6.07 (2007).

${ }^{2}$ Adoption of the Paris Agreement FCCC/CP/2015/L.9/Rev.1 (UNFCCC, 2015).

${ }^{3}$ IPCC Climate Change 2014, Impacts, Adaptation and Vulnerability, Part B, edited by C. B. Field et al. (Cambridge University Press, Cambridge, 2014).

${ }^{4}$ M. Jacobson and M. A. Delucchi, "Providing all global energy with wind, water, and solar power, Part I: Technologies, energy resources, quantities and areas of infrastructure, and materials," Energy Policy 39, 1154-1169 (2011).

${ }^{5}$ E. Marris, "Energy: Upgrading the grid," Nature 454, 570 (2008).

${ }^{6}$ J. A. Turner, “A realizable renewable energy future," Science 285, 687 (1999).

${ }^{7}$ G. Boyle, Renewable Energy (Oxford University Press, Oxford, 2004).

${ }^{8}$ F. Ueckerdt, R. Brecha, and G. Luderer, "Analyzing major challenges of wind and solar variability in power systems,” Renew. Energy 81, 1-10 (2015).

${ }^{9}$ T. Ackermann, G. Andersson, and L. Söder, "Distributed generation: A definition,” Electric Power Syst. Res. 57, 195 (2001).

${ }^{10}$ A.-M. Borbely and J. F. Kreider, Distributed Generation: The Power Paradigm for the New Millennium, Mechanical and Aerospace Engineering Series (CRC, Boca Raton, FL, 2001).
${ }^{11}$ J. C. Smith, M. R. Milligan, E. A. DeMeo, and B. Parsons, "Utility wind integration and operating impact state of the art," IEEE Trans. Power Syst. 22, 900-908 (2007).

${ }^{12}$ P. Milan, M. Wächter, and J. Peinke, “Turbulent character of wind energy,” Phys. Rev. Lett. 110, 13 (2013).

${ }^{13}$ D. Heide, L. von Bremen, M. Greiner, C. Hoffmann, M. Speckmann, and S. Bofinger, "Seasonal optimal mix of wind and solar power in a future, highly renewable Europe," Renew. Energy 35, 2483 (2010).

${ }^{14}$ D. Heide, M. Greiner, L. von Bremen, and C. Hoffmann, "Reduced storage and balancing needs in a fully renewable European power system with excess wind and solar power generation,” Renew. Energy 36, 2515 (2011).

${ }^{15}$ M. Anvari, G. Lohmann, M. Wächter, P. Milan, E. Lorenz, D. Heinemann, M. Reza Rahimi Tabar, and J. Peinke, "Short term fluctuations of wind and solar power systems,” New J. Phys. 18(6), 063027 (2016).

${ }^{16} \mathrm{M}$. Anvari, M. Wächter, and J. Peinke, "Phase locking of wind turbines leads to intermittent power production,” Europhys. Lett. 116, 60009 (2017).

${ }^{17} \mathrm{~K}$. Schmietendorf, J. Peinke, and O. Kamps, "The impact of turbulent renewable energy production on power grid stability and quality," Eur. Phys. J. B 90, 222 (2017).

${ }^{18}$ B. Schäfer, C. Beck, K. Aihara, D. Witthaut, and M. Timme, "Non-Gaussian power grid frequency fluctuations characterized by Levy-stable laws and superstatistics," Nat. Energy 3, 119 (2018).

${ }^{19}$ G. Filatrella, A. H. Nielsen, and N. F. Pedersen, "Analysis of a power grid using a Kuramoto-like model,” Eur. Phys. J. B 61(4), 485 (2008).

${ }^{20}$ M. Frasca, L. Fortuna, and A. Sarra Fiore, "A network of oscillators emulating the Italian high-voltage power grid,” Int. J. Mod. Phys. 26, 1246011 (2012).

${ }^{21}$ L. V. Gambuzza, A. Buscarino, L. Fortuna, M. Porfiri, and M. Frasca, "Analysis of dynamical robustness to noise in power grids," IEEE J. Emerg. Sel. Top. Circuits Syst. 7, 3 (2017).

${ }^{22}$ M. Rohden, A. Sorge, M. Timme, and D. Witthaut, "Self-organized synchronization in decentralized power grids," Phys. Rev. Lett. 109(6), 064101 (2012).

${ }^{23} \mathrm{M}$. Rohden, A. Sorge, D. Witthaut, and M. Timme, "Impact of network topology on synchrony of oscillatory power grids," Chaos 24, 013123 (2014).

${ }^{24}$ S. Olmi, A. Navas, S. Boccaletti, and A. Torcini, "Hysteretic transitions in the Kuramoto model with inertia,” Phys. Rev. E 90(4), 042905 (2014).

${ }^{25}$ T. Nishikawa and A. E. Motter, "Comparative analysis of existing models for power-grid synchronization,” New J. Phys. 17, 015012 (2015).

${ }^{26} \mathrm{~S}$. Olmi and A. Torcini, "Dynamics of fully coupled rotators with unimodal and bimodal frequency distribution," Control of Self-Organizing Nonlinear Systems, edited by E. Schöll, S. Klapp, and P. Hövel (Springer, Berlin, 2016).

${ }^{27}$ M. Rohden, D. Witthaut, M. Timme, and H. Meyer-Ortmanns, "Curing critical links in oscillator networks as power flow models," New J. Phys. 19, 013002 (2017).

${ }^{28} \mathrm{~L}$. Tumash, S. Olmi, and E. Schöll, "Effect of disorder and noise in shaping the dynamics of power grids," Europhys. Lett. 123, 20001 (2018).

${ }^{29}$ V. Mehrmann, R. Morandin, S. Olmi, and E. Schöll, "Qualitative stability and synchronicity analysis of power network models in port-Hamiltonian form," Chaos 28, 101102 (2018).

${ }^{30} \mathrm{H}$. Taher, S. Olmi, and E. Schöll, "Enhancing power grid synchronization and stability through time delayed feedback control," Phys. Rev. E 00, 002300 (2019).

${ }^{31}$ H. A. Tanaka, A. J. Lichtenberg, and S. Oishi, "First order phase transition resulting from finite inertia in coupled oscillator systems," Phys. Rev. Lett. 78(11), 2104 (1997).

${ }^{32}$ H. A. Tanaka, A. J. Lichtenberg, and S. Oishi, "Self-synchronization of coupled oscillators with hysteretic responses," Physica D 100(3), 279-300 (1997).

${ }^{33}$ B. Ermentrout, "An adaptive model for synchrony in the firefly Pteroptyx malaccae,” J. Math. Biol. 29, 571 (1991).

${ }^{34}$ B. R. Trees, V. Saranathan, and D. Stroud, "Synchronization in disordered Josephson junction arrays: Small-world connections and the Kuramoto model," Phys. Rev. E 71, 016215 (2005).

${ }^{35}$ P. Ji, T. K. D M. Peron, P. J. Menck, F. A. Rodrigues, and J. Kurths, "Cluster explosive synchronization in complex networks," Phys. Rev. Lett. 110, 218701 (2013).

${ }^{36}$ J. A. Acebron, L. L. Bonilla, and R. Spigler, "Synchronization in populations of globally coupled oscillators with inertial effects," Phys. Rev. E 62(3), 3437-3454 (2000). 
${ }^{37}$ R. E. Mirollo and S. H. Strogatz, "The spectrum of the locked state for the Kuramoto model of coupled oscillators," Physica D 205, 249-266 (2005).

${ }^{38}$ R. Delabays, T. Coletta, and P. Jacquod, "Multistability of phase-locking in equalfrequency Kuramoto models on planar graphs," J. Math. Phys. 58, 032703 (2017). ${ }^{39}$ D. Manik, D. Witthaut, B. Schäfer, M. Matthiae, A. Sorge, M. Rohden, E. Katifori, and M. Timme, "Supply networks: Instabilities without overload," Eur. Phys. J. Spec. Top. 223, 2527-2547 (2014).

${ }^{40} \mathrm{~T}$. Coletta and P. Jacquod, "Linear stability and the Braess paradox in coupledoscillator networks and electric power grids," Phys. Rev. E 93, 032222 (2016).

${ }^{41}$ J. Machowski, J. Bialek, and J. Bumby, Power System Dynamics: Stability and Control (John Wiley \& Sons, New York, 2011).

${ }^{42} \mathrm{~K}$. Levenberg, "A method for the solution of certain non-linear problems in least squares," Q. Appl. Math. 2, 164-168 (1944).
${ }^{43} \mathrm{D}$. Marquardt, "An algorithm for least-squares estimation of nonlinear parameters,” SIAM J. Appl. Math. 11(2), 431-441 (1963).

${ }^{44} \mathrm{~J}$. J. Moré, The Levenberg-Marquardt Algorithm: Implementation and Theory, Numerical Analysis (Springer, New York, 1978).

${ }^{45} \mathrm{The}$ map of the Italian high-voltage power grid can be seen at the web site of the Global Energy Network Institute, http://www.geni.org, and the data here employed have been extracted from the map delivered by the union for the coordination of transport of electricity (ENTSO-E), https://www.entsoe.eu/resources/grid-map/.

${ }^{46}$ B. Schäfer, M. Matthiae, X. Zhang, M. Rohden, M. Timme, and D. Witthaut, "Escape routes, weak links, and desynchronization in fluctuation-driven networks," Phys. Rev. E 95(6), 060203 (2017).

${ }^{47} \mathrm{M}$. Schröder, M. Timme, and D. Witthaut, "A universal order parameter for synchrony in networks of limit cycle oscillators," Chaos 27, 073119 (2017). 